\title{
Valutazione economica delle terapie farmacologiche
}

Lorenzo G. Mantovani ${ }^{(1)}$, Simona Cammarota ${ }^{(1)}$

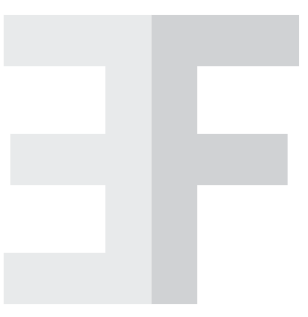

\section{FARMACOECONOMIA}

La farmacoeconomia può essere definita come la disciplina che identifica, misura e confronta i costi e i risultati dovuti ai farmaci e alle procedure terapeutiche. Va specificato che, trattandosi normalmente di terapie complesse, di cui il farmaco costituisce solo una parte, con conseguenze ed effetti che spesso trascendono il settore sanitario, il considerare il mero costo del farmaco o anche i soli costi sanitari è spesso riduttivo e inappropriato. Il problema, in questo contesto, è che la capacità di sviluppare nuove tecnologie è ancora largamente superiore alla possibilità di valutarle appieno al fine di effettuare decisioni razionali circa l'uso delle stesse. In mancanza di valutazioni adeguate e appropriate, è lecito attendersi l'assunzione di decisioni non ottimali, talora inefficienti e persino dannose.

\section{ALTERNATIVE}

Un fattore molto importante nel condurre uno studio di farmacoeconomia è rappresentato dalla scelta delle alternative terapeutiche da paragonare.

Gli studi, infatti, non vengono condotti su un solo intervento, ma su più trattamenti “omogenei". L'obiettivo di razionalizzare l'uso delle risorse richiede che i costi e gli effetti di un programma vengano paragonati con $i$ costi e gli effetti di programmi dello stesso genere, al fine di verificare quale sia il più efficiente sotto il profilo economico. Va ricordato che, perché si tratti di una valutazione farmacoeconomica, almeno una delle alternative deve essere farmacologica. Le terapie con cui il trattamento farmacologico viene confrontato possono essere anch'esse farmacologiche, oppure non farmacologiche, ma si può confrontare tale trattamento anche con l'alternativa "nessun intervento". La complessità dell'analisi aumenta man mano che si passa dalle alternative farmacologiche, a quelle non farmacologiche, al "nessun intervento", in quanto l'omogeneità tra le alternative stesse tende a diminuire. Valutare la terapia, ad esempio, con un farmaco anti- $\mathrm{H}_{2}$ rispetto a quella con un altro farmaco anti- $\mathrm{H}_{2}$ (es. cimetidina versus ranitidina) può risultare abbastanza semplice, mentre molto più complesso risulterà il confronto tra "terapia con cimetidina" e "intervento di gastrectomia", poiché sarà necessario considerare i costi di tutti gli aspetti e procedure relativi all'intervento chirurgico.

\section{PUNTO DI VISTA}

Una valutazione farmacoecononomica può essere condotta da diversi punti di vista, il più ampio dei quali è la società nel suo complesso. Esso è così vasto da comprendere tutti i possibili costi ed effetti e quindi è estremamente complesso, anche alla luce del fatto che difficilmente la società prende direttamente decisioni sull'allocazione delle risorse. La maggior parte delle valutazioni farmacoeconomiche viene, invece, eseguita dal punto di vista del terzo pagante, vale a dire dell'ente che copre la spesa sanitaria (Servizio Sanitario Nazionale, in Italia); questo perché, se la finalità dello studio deve essere quella di aiutare la decisione di allocare le risorse a un intervento piuttosto che ad un altro, il punto di vista "naturale" dell'analisi deve essere quello di chi prende tale decisione.

\section{COSTI}

Un problema fondamentale delle analisi economiche relative agli interventi farmacologici consiste nella corretta quantificazione dei costi, che dipende dalla prospettiva e dalle alternative scelte per lo studio. I costi possono essere suddivisi in: diretti (sanitari e non sanitari), indiretti e intangibili.

I costi diretti sanitari comprendono i costi dei test diagnostici, dei farmaci, delle forniture, del personale sanitario e delle attrezzature sanitarie; i costi diretti non sanitari si riferiscono, invece, direttamente all'intervento sanitario ma non sono di carattere sanitario (ad esempio i costi di trasporto del paziente). Per costi indiretti si intendono generalmente i costi derivanti dalla perdita di produttività da parte del paziente provocata dalla patologia e/o dall'intervento in esame (assenza dal lavoro temporanea o permanente). I costi intangibili, infine, riguardano tutti gli svantaggi causati dalla malattia ma che non possono essere misurati direttamente in termini quantitativi assoluti (dolore, depressione
${ }^{(1)}$ CIRFF, Centro Interdipartimentale di Ricerca in Farmacoeconomia e Farmacoutilizzazione, Università degli Studi di Napoli Federico II 
minore qualità di vita). Questi richiedono per la misurazione l'adozione di tecniche specifiche e di strumenti ad hoc.

\section{EFFETTI}

Gli effetti di un trattamento farmacologico rispecchiano la stessa suddivisione adottata per i costi (diretti, indiretti e intangibili).

Gli effetti diretti sono quelli attribuibili alle pratiche diagnostiche, terapeutiche e riabilitative connesse all'intervento in analisi o alle complicanze della patologia d'interesse. Possono essere variazioni di parametri clinici oggettivi come, ad esempio, il livello di colesterolo nel plasma o la pressione arteriosa, ma anche risultati derivanti dalla diminuzione della probabilità di determinati eventi indesiderati, quali il rischio di infarto o di altri accidenti cerebrovascolari, oppure conseguenze cosiddette finali, quali la mortalità o l'aspettativa di vita in anni. Per effetti indiretti si intendono generalmente gli effetti derivanti dalla perdita di produttività causata dalla patologia e/o dall'intervento in esame. Effetti intangibili sono tutti quelli provocati nell'individuo dal fatto di trovarsi in uno stato di non perfetta salute; non hanno carattere tangibile e non possono essere misurati direttamente, ma incidono sulla qualità di vita del paziente (ansia, stress, dolore, ecc.).

\section{IDENTIFICAZIONE, MISURAZIONE E QUANTIFICAZIONE DI COSTI ED EFFETTI}

Costi ed effetti dei programmi farmacologici devono essere identificati, misurati e quantificati economicamente. Esistono diversi metodi per rilevare e stimare gli effetti e i costi: panel di esperti, trial clinico, studio naturalistico.

La quantificazione monetaria riguarda in generale tutti i costi, mentre gli effetti vengono tradotti in unità monetarie nella sola analisi di costo beneficio, descritta in seguito. In generale i valori monetari utilizzati per la quantificazione economica devono essere coerenti con la prospettiva di analisi. Come è facile intuire, la quantificazione diviene più complessa man mano che si passa dalla categoria dei costi diretti a quella dei costi intangibili.

\section{TECNICHE DI ANALISI}

Esistono essenzialmente quattro tipologie di tecniche mediante le quali gli studi vengono eseguiti:

1. Analisi di minimizzazione dei costi o CMA (Cost Minimization Analysis): si applica per determinare il trattamento più economico nell'ambito di alternative con efficacia e finalità terapeutiche simili.

2. Analisi Costo Efficacia o ACE (Cost Effectiveness/Efficacy Analysis): misura e confronta i costi e l'efficacia, espressa in unità naturali (anni di vita salvati, morti evitate, misurazione dei livelli di colesterolo, pressione arteriosa, ecc.), relativi a interventi omogenei, vale a dire interventi che hanno effetti che possono essere quantificati con la stessa unità di misura. Ad esempio i trattamenti dell'insufficienza renale mediante dialisi o trapianto hanno in comune lo scopo di prolungare la vita del paziente. Il confronto tra i due trattamenti avverrà in base al costo per unità di effetto (costo per ciascun anno di vita salvato). L'analisi di costo efficacia è senza dubbio la tecnica più utilizzata in farmacoeconomia.

3. Analisi Costo Utilità o ACU (Cost Utility Analysis): consente di esprimere gli effetti in un parametro comune, non più monetario, bensì un valore di utilità derivato dalle preferenze degli individui o della società. Essa misura gli effetti in termini di indici di utilità: giorno in salute (Healthy Day o HD) o anno di vita guadagnato ponderato per la qualità (Quality-Adjusted Life Year o QALY). L'utilità sintetizza ed esprime, in un indice compreso tra 0 e 1, la desiderabilità di una condizione di salute, cogliendo anche gli effetti e gli aspetti intangibili, quelli cioè inerenti la qualità della vita. Per fare ciò sono necessari strumenti ad hoc di valutazione della qualità di vita. I risultati di un'analisi costo utilità sono espressi in termini di costo per HD o per QALY guadagnato a seguito della realizzazione di un trattamento rispetto a un altro. L'ACU rappresenta una tecnica più sofisticata dell' $A C E$.

4. Analisi di Costo Beneficio o ACB (Cost Benefit Analysis): si applica quando gli effetti di due o più alternative non sono direttamente paragonabili tra di loro. Ciò è possibile traducendo anche gli effetti in unità monetarie rendendoli paragonabili tra di loro e con i costi. Si richiede cioè di monetizzare i giorni di malattia evitati, gli anni di vita guadagnati, le complicanze evitate, ecc. Come è facile intuire, risulta assai difficile e talora concettualmente opinabile tradurre una vita salvata in termini di denaro. Ciò può avvenire attraverso strumenti assai complessi, come, ad esempio, la willingness to pay. Il risultato di una $\mathrm{ACB}$ viene normalmente espresso in termini di beneficio o costo netto (differenza tra costi e benefici) o di un rapporto in cui sia il numeratore (costi) che il denominatore (benefici) sono tradotti in unità monetarie. Si tratta di una tecnica ancora poco applicata in farmacoeconomia. 